\title{
Indianisation et dépolitisation des victimes de la guerre au Pérou. L'exemple du mémorial L'CEil-qui- pleure
}

Indianización y despolitización de las víctimas de la guerra en Perú. El ejemplo del memorial El Ojo que Llora

Indianization and depoliticization of the victims of the war in Peru. The Example

of The Eye that Cries memorial

\section{Dorothée Delacroix}

\section{OpenEdition}

Journals

Édition électronique

URL : https://journals.openedition.org/jsa/15186

DOI : $10.4000 /$ jsa. 15186

ISSN : 1957-7842

\section{Éditeur}

Société des américanistes

\section{Édition imprimée}

Date de publication : 15 décembre 2017

Pagination : 111-140

ISSN : 0037-9174

Référence électronique

Dorothée Delacroix, «Indianisation et dépolitisation des victimes de la guerre au Pérou. L'exemple du mémorial L'CEil-qui-pleure », Journal de la Société des américanistes [En ligne], 103-2 | 2017, mis en ligne le 15 décembre 2017, consulté le 27 septembre 2022. URL : http://journals.openedition.org/jsa/15186 ; DOI : https://doi.org/10.4000/jsa.15186 


\title{
Indianisation et dépolitisation des victimes de la guerre au Pérou. L'exemple du mémorial L'CEil-qui-pleure
}

\author{
Dorothée DeLACRoIX *
}

Le mémorial L'CEil-qui-pleure a été érigé en hommage aux victimes du conflit armé interne au Pérou (1980-2000). Il est l'œuvre de l'artiste Lika Mutal en coordination avec divers militants des droits humains et s'inscrit dans la continuité du travail effectué par la Commission de la vérité et réconciliation (CVR). L'anniversaire du rapport final de cette commission est d'ailleurs célébré sur ce site chaque 28 août. À partir de plusieurs observations de cette cérémonie, complétées par des entretiens avec l'artiste et de nombreux militants des droits humains, cet article vise à interroger le processus d'ethnicisation des victimes et la manière dont il a orienté un volet essentiel des programmes de réparation symbolique: les commémorations de la guerre. Le passé préhispanique et la « cosmologie andine » ont été mobilisés lors de ces commémorations dans le but de valoriser une catégorie particulière de victimes présentée comme innocente: les paysans quechuaphones des Andes qu'il s'agirait de « réintégrer » à la société nationale. Sont également discutés les effets sociaux et politiques dans le Pérou post-CVR de cette mise en scène de l'ethnicité qui s'appuie sur une cosmologie mystique. [Mots-clés: Pérou, conflit armé interne, monuments aux morts, mémoires de guerre, cosmologie andine, indigénisme.]

Indianización y despolitización de las víctimas de la guerra en Perú. El ejemplo del memorial El Ojo que Llora. El memorial El Ojo que llora fue construido en homenaje a las víctimas del conflicto armado interno en Perú (1980-2000). Resulta del trabajo de la artista Lika Mutal en coordinación con varios activistas de derechos humanos y se inscribe en la continuidad del trabajo realizado por la Comisión de la Verdad y Reconciliación (CVR). El aniversario del informe final de esta comisión se celebra, de hecho, en este sitio cada 28 de agosto. Este artículo, basado en varias observaciones de esta ceremonia, complementadas por entrevistas la artista y activistas de derechos humanos, tiene como objetivo examinar el proceso de etnicización de las víctimas y el modo en que ha orientado un aspecto esencial de los programas de reparación simbólica: las conmemoraciones de la guerra. El pasado prehispánico y la "cosmología andina" fueron movilizados durante estas conmemoraciones con el fin de promover una categoría particular de la víctima presentada como inocente: los

* ISPOLE, université catholique de Louvain, Belgique [dorothee.delacroix@casadevelazquez.org]. 
campesinos andinos quechua-parlantes que se deberían "reintegrar" a la sociedad nacional. También se discuten los efectos sociales y políticos en el Perú post-CVR de esta puesta en escena de la etnicidad basada en una cosmología mística. [Palabras claves: Perú, conflicto armado interno, monumentos a los muertos, memorias de guerra, cosmología andina, indigenismo.]

Indianization and depoliticization of the victims of the war in Peru. The Example of The Eye that Cries memorial. The memorial The Eye that Cries was erected as a tribute to the victims of the internal armed conflict in Peru (1980-2000). It is the work of the artist Lika Mutal in coordination with various human rights activists and is a continuation of the work done by the Truth and Reconciliation Commission (CVR in Spanish). The anniversary of the final report of this commission is also celebrated on this site every August 28th. Based on several observations of this ceremony, supplemented by interviews with the artist and human rights activists, this article aims to interrogate the process of ethnicization of victims and the way in which it has guided an essential component of symbolic reparation programs: commemorations of the war. The pre-Hispanic past and the "Andean cosmology" were mobilized during these commemorations in order to promote a particular category of victim presented as innocent: Quechuaphone peasants of the Andes who should be "reintegrated" into the national society. The social and political effects in post-CVR Peru of this staging of ethnicity based on a mystical cosmology are also discussed. [Keywords: Peru, internal armed conflict, war memorial, war memories, Andean cosmology, indigenism.]

Si on idéalise ou exalte le passé, dans ce cas le plus lointain [le passé inca], c'est justement en compensation de ce qui est nié dans le présent.

Cecilia Méndez, Incas sí, Indios no. Apuntes para el estudio del nacionalismo criollo en el Perú, 1993.

Comme dans 1'ensemble des pays latino-américains qui ont été en proie à des périodes de violences politiques durant la deuxième moitié du $\mathrm{Xx}^{\mathrm{e}}$ siècle, le Pérou connait depuis une quinzaine d'années un processus d'institutionnalisation des politiques mémorielles. Après la guerre qui opposa les forces de l'ordre au Parti communiste péruvien - Sentier lumineux (PCP-SL) - la démilitarisation du pays a été suivie d'une Commission de la vérité et réconciliation (CVR). De 2001 à 2003, elle a œuvré à la reconnaissance collective des exactions perpétrées, aux poursuites judiciaires des responsables et aux préconisations en termes de réparations, économique et symbolique, des victimes. Ces dernières ont été estimées à 70000 selon le rapport final de la CVR remis le 28 août 2003 au président de la République ${ }^{1}$. Selon ce rapport, $79 \%$ d'entre elles étaient originaires des Andes rurales du pays et $75 \%$ parlaient le quechua ou une autre

1. Comisión de la verdad y reconciliación (CVR) 2003. 
langue indigène. Dans quelle mesure ces chiffres, et les pertes humaines ainsi caractérisées d'un point de vue géographique et linguistique ${ }^{2}$, ont-ils orienté la mémoire sociale construite depuis la capitale, soit depuis le lieu de pouvoir qui n'a pas constitué l'épicentre de la guerre?

Selon l'idéologie maoïste du Sentier lumineux le soulèvement révolutionnaire devait en effet se faire depuis les campagnes. Sa stratégie militaire consista donc à instaurer des bases d'appui dans les communautés paysannes andines, lesquelles étaient considérées comme des alliées naturelles et fidèles durant le lent et sanglant processus de violence dite purificatrice contre le « vieil État réactionnaire $»^{3}$. En retour, les militaires majoritairement originaires de la côte ont d'abord perçu la paysannerie andine comme leur ennemi, avant que la stratégie gouvernementale ne tente de s'en faire un allié en imposant des milices paysannes d'auto-défense dans certaines régions du pays. À l'heure des réparations aux victimes de la guerre, supposées asseoir les bases d'une réconciliation entre les citoyens, comment et sous quels traits une figure archétypale de victime s'est-elle progressivement constituée sur la scène publique, en partie sous l'effet du travail des organismes nationaux de défense des droits humains ${ }^{4}$ ?

Pour répondre à ces questions, cet article ${ }^{5}$ se fonde sur une ethnographie des cérémonies d'hommage réalisées à Lima, plus particulièrement dans le parc du Champ-de-Mars, sur le site de L'Eil-qui-pleure. Pièce maîtresse du paysage mémoriel liménien ${ }^{6}$, ce mémorial a été inauguré le 28 août 2005 afin de célébrer le second anniversaire de la remise du rapport final de la CVR à Alejandro Toledo, alors chef de l'État. Il est constitué d'une grande pierre centrale, saillante et sombre, sur laquelle est fixé un œil d'où coule de l'eau

2. Sur le rôle de la CVR dans la mise en avant de la question indigène dans son analyse des causes et de la magnitude du conflit armé et sur les catégorisations ethniques dont elle a fait usage, influençant ainsi la caractérisation des acteurs de la guerre et les politiques de réparation aux victimes, voir Robin Azevedo et Delacroix 2017.

3. Degregori 2011; Manrique 2008; Poole et Rénique 1992.

4. L'expression «droits humains » sera préférée dans cet article dans la mesure où elle dépasse le sens masculin historiquement attribué aux «droits de l'homme » et permet de se référer explicitement aux êtres humains dans leur ensemble. Il s'agit aussi d'une traduction plus proche des expressions anglaises et espagnoles " human rigths » et " derechos humanos ».

5. Pour la pertinence de leurs remarques et leurs corrections, je tiens à remercier Capucine Boidin et Soizic Croguennec, ainsi que les deux lecteurs anonymes du Journal de la Société des américanistes. Cet article est une version remaniée et synthétisée du chapitre « La fabrique de la victime andine » issu de la version publiée de ma thèse (Delacroix 2016c, p. 71-105).

6. Premier volet du vaste projet d'aménagement intitulé « Alameda de la Memoria » (Promenade de la mémoire), l'œuvre se déploie sur un terrain de $27600 \mathrm{~m}^{2}$ cédé par la municipalité de Jesús María, un quartier de Lima. Des donations provenant de l'ambassade d'Allemagne et de Hollande, des fonds anonymes et des apports financiers complémentaires du gouvernement de Toledo ont rendu possible sa création. Le deuxième volet incluant un «Centre de dialogue et d'informations », n'a pas été avalisé depuis le changement de l'équipe municipale du quartier de Jesús María. 
évoquant des larmes. Autour se déploie un labyrinthe circulaire aux fondations arasées et composées de milliers de galets qui portent chacun le nom, l'âge et la date de disparition d'une victime de la guerre'. L'EEil-qui-pleure s'inscrit pleinement dans le cadre des recommandations de la CVR faites à l'État péruvien en termes de politiques de réparation symboliques aux victimes. Mais il résulte avant tout de donations privées et de l'engagement d'associations en faveur des droits humains afin de soutenir le travail accompli par la CVR et la diffusion de ses résultats ${ }^{8}$.

Je me concentrerai ici sur la fabrique de la victime andine à travers la monumentalité publique. La conceptualisation du mémorial et des cérémonies qui s'y tiennent manifestent en effet une certaine intellectualisation de la mémoire de guerre à Lima. Je m'attacherai à décrire la manière dont les principaux organisateurs de ces activités commémoratives ont façonné un point de vue sur la guerre et ses protagonistes. Ayant traité ailleurs de la question de la réception et des réappropriations des discours droit-de-l'hommistes dans les campagnes andines (Delacroix 2014 et 2016b), il ne s'agira pas de comprendre les représentations que les paysans andins ont d'eux-mêmes au lendemain de la guerre ni la façon dont le mémorial est perçu par ses visiteurs, mais bien d'examiner comment se construit la prédominance d'une figure de victime indianisée, souffrante et apolitique, apparaissant ainsi comme innocente à travers les hommages rendus sur ce lieu.

Les travaux qui ont pris comme objet ce mémorial se sont concentrés sur le caractère conflictuel des mémoires mobilisées dans l'espace public. Leurs auteurs ont interrogé la manière dont les victimes du Sentier lumineux et celles de l'armée se trouvent hiérarchisées dans le récit national ${ }^{9}$ (Drinot 2007, Hite 2007,

7. À l'origine 23000 noms extraits des registres de la CVR avaient été gravés sur ce mémorial. Ce nombre s'accroit au fur et à mesure des nouvelles victimes identifiées, notamment à la suite des campagnes d'exhumations de charniers.

8. Parmi les associations ayant porté ce projet nous pouvons mentionner: l'Institut de démocratie et droits humains de l'université pontificale catholique du Pérou (Instituto de Democracia y Derechos Humanos, IDEHPUCP), la Coordination nationale des droits humains (Coordinadora Nacional de Derechos Humanos, CNDDHH), le collectif citoyen Pour que cela ne se répète pas (Para que no se repita) et Association pour les droits humains (Asociación Pro Derechos Humanos, APRODEH). Des donations provenant de l'ambassade d'Allemagne et de Hollande, des apports du gouvernement de Toledo, et des fonds anonymes ont également rendu possible sa création.

9. Contrairement à d'autres pays latino-américains où la crise de légitimité des régimes dictatoriaux a favorisé une transition démocratique par la voie de la négociation (Mouchard 2000), il n'y a pas eu, au Pérou, de sortie négociée du conflit armé. La réponse de l'État vis-à-vis des groupes subversifs a été la répression via une politique de « lutte anti-subversive » qui a participé à construire les contours normatifs de l'ennemi intérieur dans l'espace social. Aujourd'hui encore, une vision dualiste des protagonistes du conflit est largement diffusée au Pérou, notamment à l'initiative des secteurs proches des militaires qui s'évertuent à héroïser 


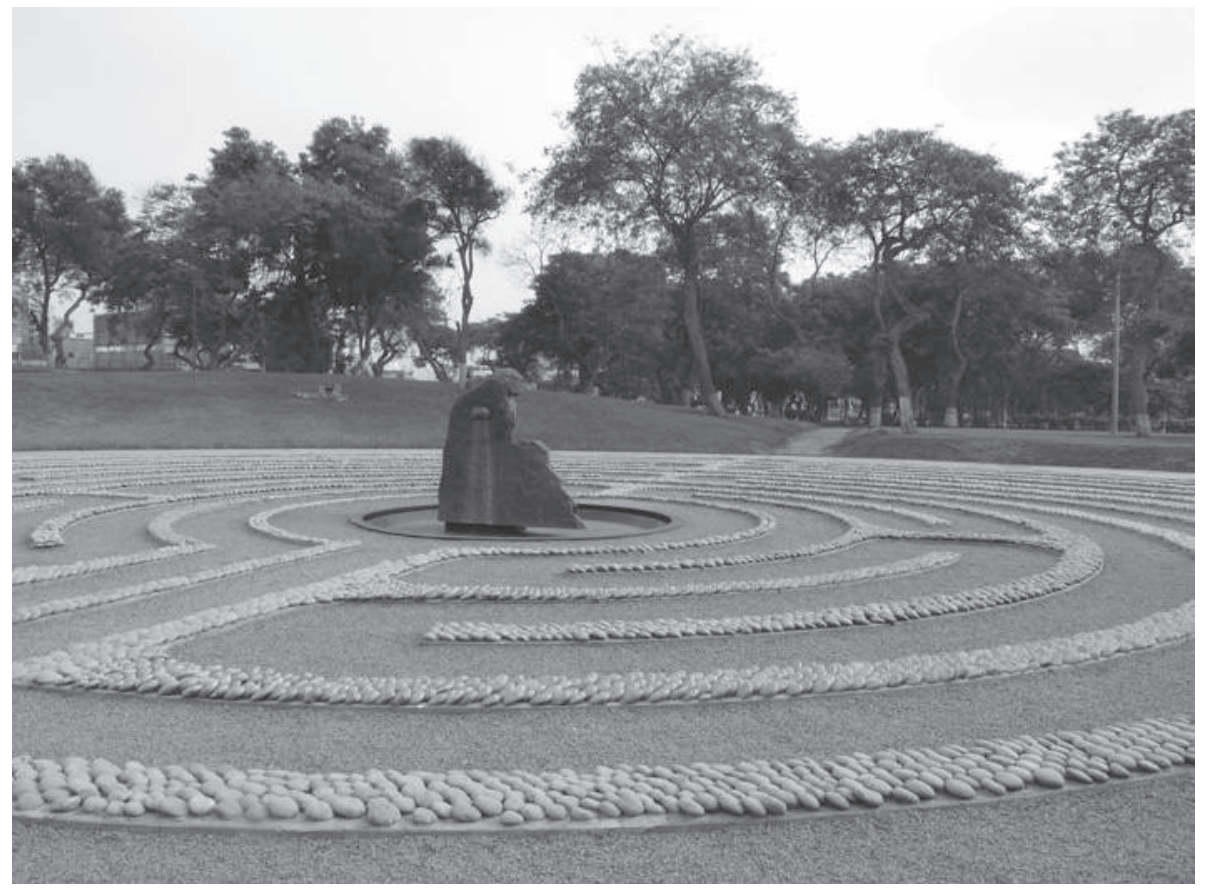

Fig. 1 - Le mémorial de L'CEil-qui-pleure dans le parc du Champ-de-Mars à Lima (photo: Dorothée Delacroix).

Milton 2011, Delacroix 2016a). En revanche, aucun ne s'est attaché à analyser la manière dont les associations péruviennes de défense des droits humains, soutenues par des organismes internationaux, véhiculent l'image d'une victime spécifiquement andine et innocente à travers les activités commémoratives organisées sur ce mémorial. Cet article cherche à comprendre les modalités, les raisons et les effets de la focalisation sur l'identité et la culture des victimes célébrées à L'EEil-qui-pleure. Pour cela, il se concentre sur la forme particulière de ritualité politique, inspirée d'un New Age andinisé, sur laquelle s'appuient les cérémonies qui y sont organisées. À travers une observation ethnographique de l'anniversaire qui se tient tous les ans le 28 août (ou aux environs de cette date) pour commémorer l'aboutissement du travail de la CVR, je montrerai la manière dont le passé préhispanique est mobilisé avant d'analyser la façon dont la « cosmologie andine » est mise au service de la mémoire de la guerre et oriente les activités commémoratives.

les membres des forces armées et de la police tout en criminalisant, voire diabolisant, les membres supposés ou réels du PCP-SL. 
Le 28 août 2005, pour la commémoration du second anniversaire de la remise du rapport final de la CVR, le mémorial de L'Eil-qui-pleure est inauguré dans le parc du Champ-de-Mars de Lima. En 2009 et 2011, les cérémonies commémoratives du 28 août se sont déroulées sur un modèle très similaire. Le parc du Champ-de-Mars a été investi par des danseurs richement vêtus, aux gigantesques coiffes ornées de plumes. Ils se sont dirigés vers le mémorial de L'CEil-qui-pleure. Des effluves d'encens envahissaient 1'air. On distinguait l'odeur caractéristique du palo santo (Bulnesia sarmientoi), ce bois utilisé en Amazonie lors des rituels chamaniques pour ses propriétés purificatrices et sa capacité à éloigner les mauvais esprits. Des milliers de bougies illuminaient le site. Certaines étaient placées devant la photo d'une victime. Plus encore que leurs noms inscrits sur les galets du mémorial, ces portraits utilisés lors des commémorations les individualisent et les incarnent. Les participants à la cérémonie évoluaient ainsi à travers les représentations de celles et ceux qui ont disparu durant le conflit armé. Silencieusement, une procession parcourut le labyrinthe dans une attitude de recueillement. En atteignant le bassin qui entoure la roche centrale, certains se sont agenouillés et signés avec l'eau du bassin. Ces allées et venues dans le labyrinthe étaient rythmées par des chants religieux en quechua interprétés par une troupe de théâtre liménienne dénommée Yuyachkani ${ }^{10}$. Les fleurs déposées auprès d'un nom recouvraient bientôt le site gris d'une multitude de points colorés. Au pied de la sculpture centrale trônait une gerbe de fleurs. Sur les collines artificielles qui entourent le mémorial ${ }^{11}$, des dizaines d'associations de familles de victimes formaient une gigantesque ronde, certains se donnaient la main, d'autres brandissaient des banderoles sur le thème du « plus jamais ça » (nunca más). La fumée dégagée par les encens et le palo santo envahissait le site en créant une atmosphère de recueillement. L'observateur de cette cérémonie pouvait avoir l'impression que les participants partageaient un sentiment de commisération profonde, éventuellement pouvaitil juger que des éléments disparates s'entrechoquaient mais convergeaient à célébrer une victime souffrante et à appeler au soulagement de sa douleur.

10. En quechua, yuyachkani signifie « je me souviens ».

11. Ces collines, construites par un architecte qui a travaillé avec la sculptrice de l'œuvre, permettent d'isoler le mémorial de l'avenue adjacente qui est très passante. Reste que la présence récurrente d'une fête foraine dans l'enceinte du Champ-de-Mars - et à proximité immédiate de L'Eil-qui-pleure - ainsi que le parc canin contiguë au mémorial, sont des éléments qui tendent à asseoir l'idée d'un lieu de recueillement impossible. Du reste, l'accès au mémorial est extrêmement restreint. En raison des saccages successifs qu'il a connus en 2007, il a été fermé au public et finalement clôturé par un grillage en 2012. 


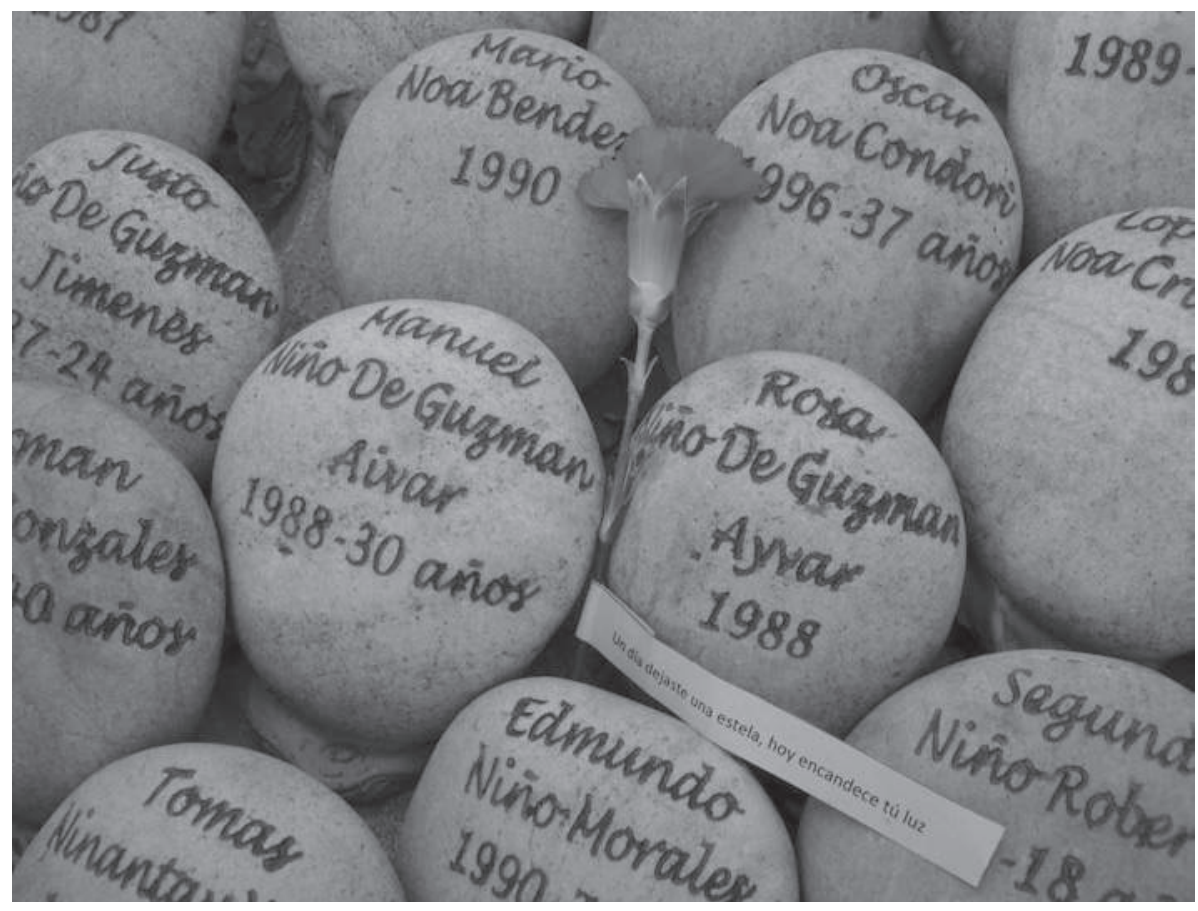

Fig. 2 - Détail du mémorial de L'CEil-qui-pleure (photo: Dorothée Delacroix).

\section{L’inauguration du mémorial : un passé incaïque magnifié}

Cette inauguration a tout d'abord permis aux militants ${ }^{12}$ de se doter d'un site particulier de rassemblement. Auparavant, des manifestations polymorphes se déroulaient tant à Lima qu'à Ayacucho ${ }^{13}$. Or, la manière dont a été pensée la

12. Pour éviter la répétition de la formule « militants en faveur des droits de l'homme », j'utiliserai les termes de « militants » et d' « acteurs associatifs » pour me référer, de façon générique, aux individus mobilisés pour les droits humains, qu'ils soient employés par des ONG ou bénévoles, en tout cas toujours organisés au sein d'un réseau plus large, et participants aux manifestations.

13. L'inauguration, par les membres de la CVR, d'une plaque en hommage aux victimes à Ayacucho le 29 août 2003 (soit le lendemain de la remise du rapport final au président de la République) et le concert de musique péruvienne qui suivit marquent le premier jalon d'une politique mémorielle qui mêle spectacle culturel et engagement en faveur des droits humains. L'inauguration de L'Eil-qui-pleure, deux ans plus tard, s'inscrit dans ce même objectif. Elle avait réuni des danseurs de ciseaux (danzantes de tijeras), caractéristiques de la région d'Ayacucho, le groupe de rock péruvien Mar de Copas, le chanteur de folklore andin William Luna et le groupe de théâtre Yuyachkani (composé majoritairement d'intellectuels liméniens). Du concert tendance à l'action citoyenne de revendication des droits humains, l'éclectisme était de mise. 
cérémonie inaugurale est tout à fait éclairante du travail d'intellectualisation de la mémoire de la guerre effectué par les secteurs militants de la capitale ${ }^{14}$. La présentation publique de L'Eil-qui-pleure en 2005 s'inscrivait dans le cadre d'une campagne nationale de diffusion des résultats de la CVR organisée par deux institutions publiques et par une association de défense des droits humains ${ }^{15}$. Ces trois organismes, composés quasi exclusivement de Péruviens issus de l'élite intellectuelle du pays ${ }^{16}$, estimaient qu'une stratégie novatrice était nécessaire afin d'expliquer l'importance du rapport final de la CVR d'une part, et de faire en sorte que des politiques publiques puissent être mises en œuvre d'autre part. Pour ce faire, une gigantesque activité baptisée « Caminata por La Paz y la Solidaridad » (la marche pour la paix et la solidarité) a été déployée à travers le pays sur $2350 \mathrm{~km}$, de la frontière nord avec l'Équateur à la frontière sud avec la Bolivie. Ce parcours, au fil de ses étapes, avait pour objectif la construction du Gran Quipu de la Memoria (Grand quipu de la mémoire).

Il faut revenir à la période préhispanique, lorsque les Andes étaient sous domination inca (entre le début du Xv $\mathrm{XV}^{\mathrm{e}}$ siècle et 1532 ), pour comprendre la réutilisation qui est faite en 2005 d'un élément emblématique de cette culture. Le quipu est un système d'écriture composé de cordelettes de coton ou de laine de différentes couleurs comportant des nœuds. Il servait principalement pour la comptabilité, et a pu être doté d'un véritable contenu narratif à travers « une écriture logographique comparable aux écritures hiéroglyphiques égyptienne ou maya » (Itier 2010, p. 147) ${ }^{17}$. Cet élément est réintroduit dans la commémoration de la guerre civile d'une manière particulière : chaque nœud représente une vie perdue durant le conflit. Le projet mobilise des milliers de citoyens à travers le pays, qui réalisent ces nœuds et les transmettent d'une ville à une autre.

De mai à août 2005, des bénévoles ont sillonné « le chemin pour la paix et la solidarité » sur les vestiges du Qhapaq ñan (route royale), l'ancien réseau routier inca. Ils étaient appelés chaski, un terme qui désignait à l'origine le messager chargé de parcourir des kilomètres en courant pour transmettre des messages ou

14. Bien qu'absente lors de cette cérémonie de 2005, j'ai pu recueillir des documents de travail produits par diverses associations, ainsi que des publicités réalisées sur l'événement. J'ai aussi pu visionner les vidéos mises en ligne par les associations. Ce matériel a été complété, à partir de 2009, par des entretiens avec des personnes qui ont participé à cette activité. Les cérémonies d'anniversaire de L'CEil-qui-pleure ont ensuite fait l'objet d'observations ethnographiques répétées (en 2009, 2011 et 2012).

15. Les deux institutions publiques sont la Table de concertation pour la lutte contre la pauvreté (Mesa de concertación para la lucha contra la pobreza) et le médiateur de la République (Defensoría del pueblo). Elles travaillent en collaboration avec le collectif citoyen Pour que cela ne se répète pas (Para que no se repita).

16. Diplômés d'études universitaires jusqu'à la Licence ou la Maîtrise, ils sont originaires des grandes villes du pays et ne parlent pas le quechua.

17. Voir aussi Salomon 2004 et Brokaw 2010 pour une synthèse des interprétations existantes au sujet des quipus et de leurs usages. 
des petits objets à l'époque des Incas. Alors que les chaski portaient des sandales de cuir, le prospectus publicitaire de cette campagne nationale montre des baskets entachées de sang. À côté de cette image, on peut lire: « tes pas revivront 69280 histoires » en allusion à l'estimation du nombre de victimes fournie par la CVR et au nombre de nœuds sur les quipus qui devait être atteint. Cette activité a été conçue pour susciter la participation des habitants en les sensibilisant tant au passé incaïque qu'à celui de la guerre civile (Jallade 2011). Ce grand road-movie de la mémoire de la guerre s'est clôturé par l'inauguration de L'CEil-qui-pleure. Les chaski sont entrés dans la capitale le 26 août 2005 au soir devant plus de 15000 personnes en brandissant des drapeaux arc-en-ciel, emblème moderne de l'Empire inca. Ils étaient accompagnés par un défilé d'organisations de familles de victimes. Pourquoi faire le choix de ces références foisonnantes à l'époque préhispanique comme mode de traitement mémoriel de la guerre?

Les populations andines quechuaphones ont été les plus affectées par le conflit armé. Le bilan dressé par la CVR a révélé des pertes humaines trois fois plus lourdes que ce qui avait été jusqu'alors imaginé ${ }^{18}$. Le caractère vertigineux de ces chiffres a obligé les élites urbaines à réaliser qu'elles n'avaient pas vu disparaître ses concitoyens et a alimenté chez elles une dette morale à l'égard des paysans andins. Rosario Narváez Vargas occupe un confortable appartement situé au dernier étage d'un immeuble récent de l'élégant quartier de Pueblo Libre. Elle est âgée de moins de 60 ans et a fait des études universitaires en sciences sociales. Rosario s'est investie au sein de l'Association pour les droits humains (Asociación pro derechos humanos, APRODEH) dès 1985, soit deux ans après sa création ${ }^{19}$. Rapidement employée par cette $\mathrm{ONG}$, elle est désormais « directrice de coordination et de gestion de l'information ». Son travail consiste notamment à organiser les manifestations sur le site de L'Eil-qui-pleure. Elle fait figure de tête pensante du déroulement de la cérémonie du 28 août en relation avec les autres associations participantes. Elle explique en ces termes la dette morale des Liméniens à l'égard des « Andins ${ }^{20} »$ :

18. Sur les presque 70000 victimes du conflit armé, le rapport final estime que $79 \%$ d'entre elles vivaient en zone rurale et que $75 \%$ avaient pour langue maternelle le quechua ou une autre langue indigène alors que ces locuteurs ne représentaient que $16 \%$ de la population totale du pays ("Conclusiones generales », in CVR 2003, p. 316). Le bilan de la CVR est un choc pour le pays car jusque-là les chiffres de 20000 à 25000 morts et disparus étaient avancés.

19. L'APRODEH est la principale association œuvrant pour les droits humains au Pérou. Elle a été créée en 1983 par Francisco Soberón qui en est encore à la tête aujourd'hui. L'association reçoit notamment le soutien de la Fédération internationale des droits de l'Homme (FIDH), du Secours catholique et de Oxfam (en Grande-Bretagne). L'APRODEH a ouvert des antennes en province, notamment à Abancay en Apurímac.

20. L'emploi par les militants des droits humains de l'ethnonyme « Andin » est courant, pour ne pas dire systématique. J'utilise des guillemets pour m'y référer en tant qu'hétérodésignation car les paysans des Andes ne s'identifient pas de la sorte, préférant une identité basée sur leur catégorie socio-professionnelle ou leur village d'origine (Robin Azevedo 2004a). 
Les Liméniens qui ont un certain niveau de sensibilité avec le thème [du conflit armé], ont une dette, sentent une dette, envers les victimes des Andes en particulier, qui ont été les victimes majoritaires. Qu'il y ait donc, à Lima, des expressions d'hommage aux victimes à un niveau national et que celles-ci reprennent des éléments du monde andin, moi ça me semble faire partie de cette reconnaissance à laquelle on veut arriver pour tenter de comprendre des éléments d'une culture que j'apprécie, respecte et connais, mais qui m'est étrangère. (Entretien avec Rosario Narváez Vargas, Lima, le 20 juin 2009)

En termes commémoratifs, ce sentiment de dette a eu pour effet la focalisation des activités mémorielles organisées depuis 2003 sur les populations andines. Or c'est une andinité pétrie de survivances incaïques qui est donnée à voir car, aux yeux des militants en faveur des droits humains, la filiation suggérée entre l'Empire inca et la société andine actuelle participerait d'un effort de valorisation de cette dernière. De prime abord, cette position semble similaire à celle des néo-Indiens qui, dans leur quête d'autochtonie et d'énergies cosmiques, n'hésitent pas à faire feu de tout bois, puisant aussi bien dans les représentations idéalisées de 1'Empire inca que dans les rituels décrits par les ethnologues. De cette manière, ils donnent forme à un « télescopage tellurique entre un passé glorieux inventé et un avenir radieux » (Galinier et Moliné 2006, p. 9). Dans le cas étudié, le recours à l'incaïsme et, on le verra, à la cosmologie andine, prétend servir de fondement à un avenir pacifié et appelle, pour l'atteindre, à un mouvement politique articulé, lequel dépasse le cadre strictement spirituel du néo-indianisme.

Moi je le vois comme une revendication. Le Grand quipu de la mémoire me paraît intéressant, il lie le passé lointain des Incas avec le passé récent. Aussi l'usage des quipus [...] m'a paru sympathique, [il permet] aux jeunes de se souvenir de l'usage historique des quipus par le biais d'un thème actuel. Il me semble que cela a été une campagne importante. Moi je crois qu'elle a pour fondement de revendiquer des éléments de la culture andine comme une reconnaissance vers une meilleure considération. (Entretien avec Rosario Narváez Vargas, Lima, le 26 août 2009)

Cette mise en scène d'un passé lointain, pour glorieux qu'il soit, a pourtant des effets paradoxaux. Rebâtir du commun entre les citoyens en puisant dans les racines préhispaniques du pays a pour effet de passer sous silence la violence structurelle de la société péruvienne actuelle. En effet, la scénographie des commémorations du conflit armé, en mobilisant une kyrielle de symboles de l'Empire inca, se détourne du propos initial, celui d'évoquer la guerre et ses principales victimes originaires des Andes rurales qui vivent en situation de marginalisation sociale. La filiation suggérée entre les Incas et la société andine contemporaine contribue à réifier ces populations et à les éloigner de la modernité. Les victimes andines commémorées à Lima le sont sous l'angle d'une altérité de l'intérieur. Cette perspective contribue à une mise à distance des locuteurs quechua paysans perçus comme profondément différents du reste de la population. 
Par ailleurs, contrairement à de nombreux autres pays latino-américains, le Pérou n'a pas connu de mouvements indianistes ayant eu un réel impact sur la société, excepté en Amazonie avec l'Association interethnique de développement de la forêt [amazonienne] péruvienne (Asociación interétnica de desarollo de la selva peruana, AIDESEP). La catégorie ethnique d' " Indien » pour désigner les populations andines est donc quasi exclusivement assignée de l'extérieur soit pour glorifier, soit pour discréditer ces populations. Elle est mobilisée positivement par les acteurs internationaux et les ONG de défense des droits humains sans pour autant faire l'objet d'une réappropriation par les principaux intéressés. Pour la plupart, ces derniers s'identifient plus spontanément à une identité locale liée à leur village d'origine, tout en se disant avant tout Péruviens, ce qui laisse transparaître le souhait d'être considérés comme des citoyens lambda. On constate par exemple que les cérémonies commémoratives de la guerre réalisées dans les Andes, notamment en Apurímac, participent d'une revendication du statut de citoyen à travers la mobilisation systématique de symboles nationaux tels que le lever de drapeau et le chant de l'hymne national (Delacroix 2014).

\section{Usages d'une indianité envisagée de l'extérieur}

Afin de rendre compte de l'existence de statuts et d'assignations identitaires fortement cloisonnés au Pérou, il est nécessaire de rappeler brièvement l'origine des catégorisations ethniques, qui, bien qu'ayant connu maintes transformations, demeurent un cadre de représentation difficilement dépassé.

Le contexte de décolonisation et d'indépendance du Pérou en 1824 a paradoxalement renforcé la discrimination des populations indiennes à travers un durcissement des hiérarchies sociales entre Indiens et Créoles. En effet, comme l'a montré Cecilia Méndez (1993), l'existence même d'une noblesse indigène à l'époque coloniale empêchait toute considération de l'Indien comme un être absolument subalterne. Or les indépendances ont entraîné la paupérisation et le déclassement de l'aristocratie indienne à travers la suppression des rares privilèges qui lui étaient associés et de toute différenciation socio-économique et symbolique parmi la catégorie d' «Indien ». Cette situation a impliqué le nivellement par le bas de ceux qui étaient considérés comme tels. Marginalisés économiquement, juridiquement et symboliquement, ils ont perdu tout « intellectuel organique », pour reprendre les termes de Gramsci, qui aurait été susceptible de les représenter parmi les élites nationales. Et ce en particulier après l'abolition des caciques déclarée en 1825 par Bolivar. De façon contradictoire vis-à-vis de l'idéal bolivarien, c'est au moment où l'Indien se convertit en un citoyen péruvien et où se concrétise un vaste mouvement de privatisation foncière, censé favoriser une classe de petits producteurs indépendants, que prend racine un « néocolonialisme interne » (Favre 1996, p. 28, et Piel 1975). En outre, les Créoles s'étaient déjà arrogés à l'époque coloniale la rhétorique 
de la glorification du passé inca dans l'objectif de réduire à néant toute velléité de revendication identitaire de la part des populations indigènes, surtout au lendemain de la révolte de Tupac Amaru (1780-1781) ${ }^{21}$.

En s'appropriant et en officialisant un discours qui à l'origine appartenait à l'aristocratie indigène, les créoles neutralisaient le sens politique qu'auraient pu avoir ces expressions propres aux Indiens. En outre, faire appel aux glorieux incas, réels ou imaginés, pour défendre le Pérou d'une invasion était une manière d'établir le caractère « déjà donné » de la nationalité et de nier la possibilité que celle-ci ait été forgée depuis les secteurs indigènes, les métis et la plèbe. (Cecilia Méndez 1993, p. 24, traduction de l'auteur)

Faire des Incas les ancêtres des Péruviens tout en ségréguant les Indiens a constitué un discours au fondement de la République aristocratique (18951919). Cette récupération à des fins politiques du passé inca comme ancêtre mythique fondateur de la nation excluait de fait les Indiens « en chair et en os ». La question de la place de « l'Indien » dans le processus de construction nationale apparaît après la guerre du Pacifique $(1879-1883)^{22}$. Elle se traduit par des discours indigénistes animés d'une volonté de défense du passé indigène et d'intégration nationale de « l'Indien » dans une perspective protectionniste. Dès le début $\mathrm{du} \mathrm{XX}^{\mathrm{e}}$ siècle, diverses associations pro-indigènes naissent à l'initiative des jeunes générations, avec l'ambition de valoriser les racines autochtones dans le but de donner à la nation sa spécificité propre ${ }^{23}$. Ceci en opposition à l'hispanisme dominant au sein des élites. Mais ces discours sur « l'Indien » sont émis par des intellectuels urbains peu au fait des conditions de vie quotidiennes dans les Andes rurales.

Le mouvement indigéniste est la manifestation, non d'une pensée indienne, mais d'une réflexion créole et métisse sur l'Indien. Il se présente d'ailleurs comme tel, sans jamais prétendre parler au nom de la population indienne. Il n'empêche qu'il décide de son sort en ses lieux et place, selon les intérêts supérieurs de la nation tels que les indigénistes les conçoivent. (Favre 1996, p. 6)

À l'aune de ces considérations historiques, il est possible de mieux saisir les tentatives contemporaines de réhabilitation de la paysannerie andine au lendemain de la guerre. Alors que le mouvement indigéniste est né à un moment où le Pérou cherchait à se constituer en nation, le pays a besoin de recomposer cette unité nationale à la suite de la guerre. Comme l'a soutenu Anne-Marie Thiesse (1999, p. 14), « la nation naît d'un postulat et d'une invention. Mais elle ne vit que par l'adhésion collective à cette fiction ». Or les vingt années de conflit armé interne qu'a connu le pays, et qui a laissé le plus lourd bilan de

21. Sur ce point, voir Walker 2015.

22. Cette préoccupation s'accentue ensuite durant les deux premières décennies du $\mathrm{xx}^{\mathrm{e}}$ siècle.

23. Sur cette période dans le Cuzco, voir notamment: Francke Ballve (1980) et Rénique (1991), 
l'histoire militaire du pays depuis son Indépendance (CVR 2003), a mis à mal l'idée même d'appartenance à un collectif national. Pendant la guerre civile, la racialisation polarisée de la société péruvienne s'est hypertrophiée. Les habitants de la côte qui alimentent le gros des troupes de l'armée ont d'abord perçu le paysan andin comme un Indien, synonyme d'arriération, et comme l'ennemi naturel des militaires. Ils l'ont confondu avec la figure du terroriste avant que l'armée ne crée des milices paysannes d'auto-défense.

Inévitablement, le défi de reconstituer un socle commun après ce conflit fratricide et de réhabiliter la figure du paysan andin se pose de façon cruciale. Le passé préhispanique, extrêmement valorisé par la société péruvienne ${ }^{24}$, offre justement une base d'identification commune d'autant plus utile qu'elle est apolitique. Ainsi s'explique la démarche qui consiste à lier des éléments emblématiques de ce passé lointain mais noble avec la culture andine actuelle. « L'Andin » que les militants des droits humains donnent à voir est le dépositaire de toutes les valeurs positives et la victime innocente de l'histoire de la conquête et de la guerre civile. Loin du cliché du paysan pauvre et révolutionnaire, c'est un indigène paré de ses plus beaux habits qui déambule sur le site du mémorial de Lima et qui est admiré de tous.

\section{Les danseurs Sicuris : une andinité recrée}

Le 28 août 2009, des danseurs ornés de plumes et d'habits richement colorés ont accompagné la Manifestation pour la justice et la vérité précédant la quatrième cérémonie d'anniversaire de L'CEil-qui-pleure organisée par les ONG et les organisations de défense des droits humains (Figure 3). Il s'agissait de danseurs Sicuris, emblématiques des hauts plateaux de la région de Puno, qui ont investi le mémorial avant les premiers discours. Toutefois, ces danseurs étaient issus d'un groupe folklorique de Barranco, un quartier tendance de Lima très fréquenté par les artistes.

Cette présentation publique vient asseoir une figure de «l'Andin » avec ses plumes et sa longue flûte, largement fantasmée dans les représentations de certains Liméniens et, au-delà, dans l'imaginaire occidental. Cette mise en scène rappelle le point de vue développé par Henri Favre (2009) selon lequel une ONG fonctionne à l'instar d'une entreprise privée dans le cadre de l'économie capitaliste, produisant des causes humanitaires, environnementales ou autres et les commercialisant sur le marché. On retiendra de cette analogie provocatrice la fabrique fantasmée et stéréotypée de la figure de l'indigène par les $\mathrm{ONG}$ afin de répondre à l'imaginaire occidental et à sa demande. À Lima, les militants des droits humains manient cette image de l'altérité indienne afin

24. Voir notamment Riviale 2001. 


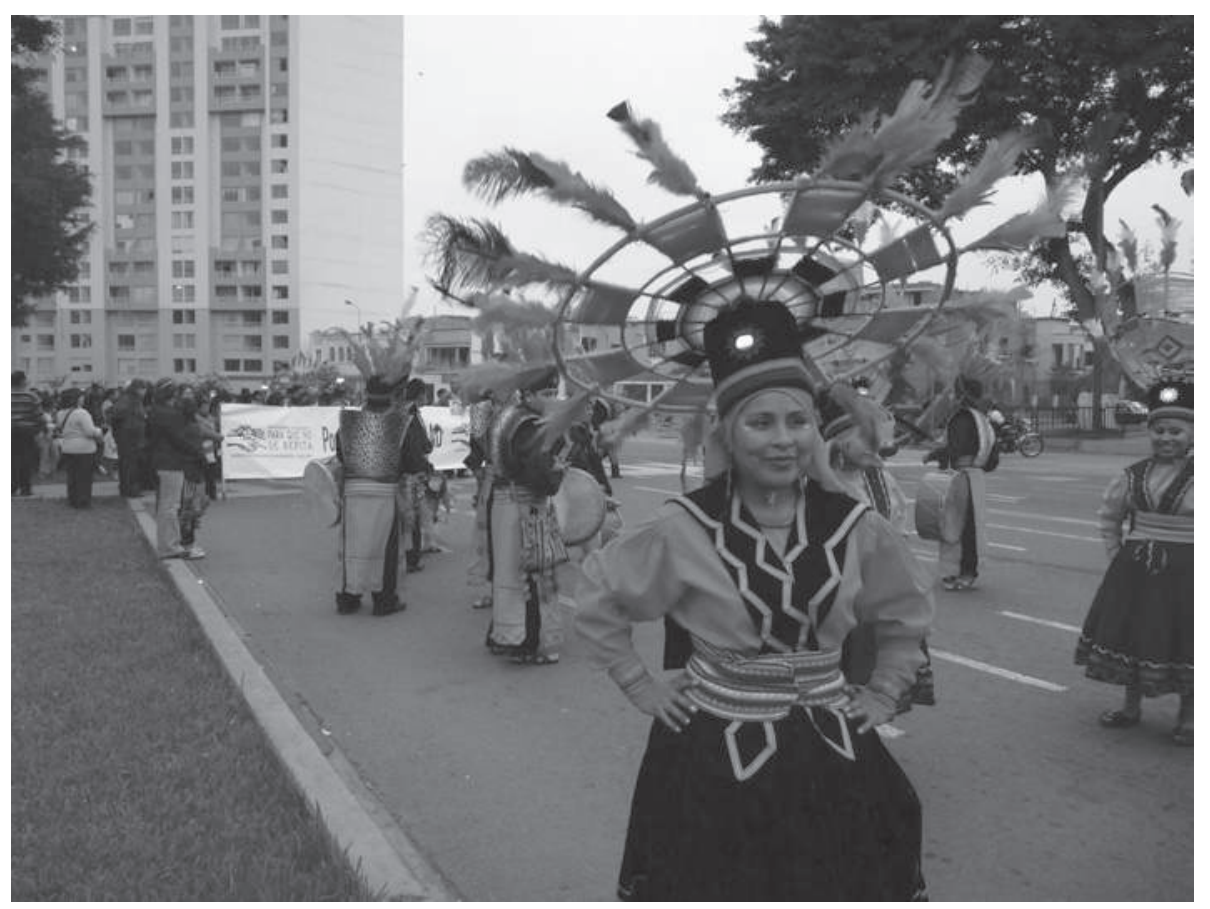

Fig. 3 - Danseurs Sicuris aux abords du parc du Champ-de-Mars.

Ils accompagnent la manifestation qui a précédé la cérémonie du 28 août 2009 sur le site de L'Eil-qui-pleure (photo: Dorothée Delacroix).

de sensibiliser à la situation sur la question des droits humains et à la situation post-conflit pour les victimes, majoritairement paysannes et quechuaphones.

Dans un effort de reconnaissance des populations les plus touchées par la guerre, on assiste à un processus d'ethnicisation de la mémoire qui se traduit par une volonté de se référer à toute une série de traits culturels andins tels que la danse, le quechua ou encore, on le verra, une forme de religiosité et un rapport à la nature spécifiques. De façon emblématique, le quechua qui était et est toujours objet de mépris lorsqu'il est parlé dans la capitale, est devenu un élément aussi essentiel que folklorisant des commémorations de la guerre. Karine Vanthuyne a analysé un processus similaire de re-ethnicisation maya à l'œuvre dans le Guatemala post-conflit ${ }^{25}$. Elle explique que l'objectif du Centre d'action légale pour la défense des droits humains (Centro para la acción legal en derechos humanos, CALDH) de poursuivre juridiquement le haut commandement militaire pour crime de génocide « en face d'un gouvernement qui refuse d'admettre cette définition du conflit, [a conduit] cette organisation à mettre

25. Voir Vanthuyne 2014. 
l'accent sur une identité commune de Maya » (Vanthuyne 2014, p. 142-143). L'auteure décrit l'une des réunions semestrielles organisées par le CALDH en mars 2004.

Un prêtre maya, Miguel, dont le CALDH avait sollicité les services spécialement pour [cette réunion], célébra une « cérémonie maya ». Accroupi sur le sol, au milieu d'un site avoisinant l'autel où avait lieu [la réunion] il commença la cérémonie en traçant avec du sucre un cercle sur le sol, qu'il divisa ensuite en quatre parties. " Ces quarts de cercles représentent les quatre points cardinaux », expliqua-t-il en espagnol aux quelques Achi, Américains, Anglais, Autrichiens, Canadiens, Chuj, Espagnols, Français, Kaqchikel, K'iche, Mam, Q'anjob'al, Q'eqchi' et Suédois qui s'étaient rassemblés autour de lui pour assister à la cérémonie. Puis, dans chacun de ces quarts de cercles, Miguel disposa des boulettes de résines et des copeaux de copal, de l'encens et des chandelles, qu'il arrosa ensuite d'alcool et de jus. Expliquant qu'il s'agissait d'offrandes à la terre, il y mit le feu. Après quelques prières en mam prononcées à voix basse, Miguel invita, à voix haute en espagnol, chacun des participants à la cérémonie à venir se purifier dans la fumée. Pendant que nous défilions dans la fumée, Miguel poursuivit la cérémonie en espagnol demandant à la terre sa bénédiction, et ce, non seulement en abondance de pluie et de maïs, mais aussi pour le long chemin de la justice. [...] Miguel insista sur la nécessité pour le Peuple maya de rester uni. Bien que de religions différentes, tous adoraient le même Dieu. Et pour avancer sur le long chemin de la justice, il fallait qu'ils cheminent non pas seuls mais tous ensemble, sinon ils n'obtiendraient strictement rien. (Vanthuyne 2014, p. 145-146)

L'accent mis sur l'indigénéité des victimes au Pérou comme au Guatemala relève pourtant de stratégies politiques différentes, tant de la part des organismes des droits humains qui les encouragent, que des survivants. En effet, au Guatemala, l'ethnicisation des victimes s'est doublée d'une re-politisation de celles-ci. Elles ont été encouragées à inscrire leur expérience du conflit armé « dans la longue histoire des rapports d'exploitation, de domination et d'oppression qui les ont précédés et suivis » (Vanthuyne 2014, p. 135). Afin de mobiliser les paysans de l'Altiplano guatémaltèque pour la justice, il était dans l'intérêt du CALDH de renforcer la conscientisation des survivants en tant que sujets politiques et sujets de droit. Au Pérou, cette étape de re-politisation publique des mémoires de la guerre n'est pas à l'ordre du jour ${ }^{26}$. On lui préfère une valorisation de l'identité ethnique des Andins, dont le statut de victimes passives est mis en avant. Cette insistance sur l'ethnicité des victimes, contrairement au cas

26. À titre d'explication, nous pouvons émettre l'hypothèse que les discours politisés des anciens guérilleros au Guatemala ne sont pas aussi tabous que ceux des sentiéristes dans la mesure où les premiers n'ont causé que très peu de victimes, contrairement au Sentier Lumineux. La Commission guatémaltèque rapporte en effet que $3 \%$ du nombre total des victimes (estimé à 200000) serait le fait des groupes insurgés (Comisión para el Esclarecimiento Histórico 1999, p. 26) contre $54 \%$ au Pérou. Ces mouvements révolutionnaires ne sont donc pas entourés de la même condamnation sociale. 
guatémaltèque, ne fait l'objet ni d'une réappropriation ni d'une identification de la part des principaux concernés ${ }^{27}$. En conséquence, les revendications formulées par les militants liméniens au nom des paysans andins sur la base de leur supposée indianité participent d'une réactualisation du paradigme indigéniste. On pourrait ajouter que la référence au paysan pauvre des Andes centrales péruviennes n'étant pas assez vendeuse, il était nécessaire que la victime célébrée le 28 août corresponde, au moins en partie, aux canons de l'indigène tels qu'ils sont représentés sur la scène internationale. Au sein du dispositif sémantique du mémorial, l'emphase mise sur le paysan andin alimente finalement une certaine rigidification des identités, illustrant parfaitement le constat de Tzvetan Todorov (1993, p. 34) selon lequel « le culte de la mémoire [...] peut être l'expression du conservatisme et de la survalorisation de l'identité ».

\section{Utilisation de la « cosmologie andine ». Réinvention de la figure de la Pachamama}

La pierre centrale de L'Eil-qui-pleure est le pivot d'un discours d'intellectualisation de la cosmologie andine, laquelle est postulée comme une caractéristique majeure du rapport au monde des personnes originaires des Andes. Cette représentation est en partie liée au mysticisme New Age et plus spécifiquement au mouvement néo-indien péruvien qui lui est antérieur (Galinier et Molinié 2006). Celui-ci se caractérise par une démarche spiritualisante soutenue par une quête d'autochtonie qui, paradoxalement, ne connait pas de frontières. S'inscrivent dans cette mouvance tant des artistes liméniens que des touristes étrangers. C'est le cas de Lika Mutal ${ }^{28}$, sculptrice hollandaise installée depuis trente ans à Lima, qui a été l'instigatrice du projet du mémorial de L'Eil-qui-pleure. Elle exprime sa relation à la pierre en ces termes:

27. D'une part, les populations andines veulent éviter d'être associées au stigmate de « l'Indien subversif » dans un contexte où l'État, vainqueur de la guerre, octroie des réparations économiques aux victimes uniquement si celles-ci n'ont pas appartenues aux guérillas. L'exclusion des membres de groupes subversifs du Registre unique de victimes (RUV) est en effet fixée par l'article 4 de la loi 28592 (Ley que crea el Plan integral de reparaciones [PIR]). D'autre part, la catégorie ethnique d'Indien a historiquement été rejetée par les populations andines, en particulier sous le gouvernement militaire de Velasco Alvarado (1968-1975) qui a supprimé cette catégorie au profit de celle de paysan afin de tenter de dépasser les divisions ethnico-raciales de la société péruvienne.

28. Lika Mutal (1939-2016) est née en Hollande dans une famille d'artistes (sa mère est musicienne, son père artiste peintre). Après avoir étudié les beaux-arts à Utrecht et Bogotá, elle arrive à Lima en 1968 et entreprend une formation à la sculpture à l'université pontificale catholique du Pérou (Pontificia Universidad Católica del Perú). En 1970, elle obtient le premier prix de sculpture de cette université. Elle vit à New-York et y expose à plusieurs reprises, avant de s'installer définitivement au Pérou en 1984. 
Nous les artistes, nous connaissons la résistance nécessaire pour que l'idée créatrice se manifeste de façon claire, mais nous savons qu'une fois à l'œuvre nous devenons les artisans de quelque chose qui prend le contrôle, qui amplifie notre idée. Dans le cas de la pierre, j'ai toujours soupçonné qu'elle-même porte une idée, qu'elle veut s'exprimer et que l'espace est inhérent à l'œuvre. Pour ceux qui appartiennent à la culture occidentale, cela semble bizarre parce que, selon la logique rationnelle, la pierre n'est qu'un matériau et que la dimension animique lui est niée. Or au Pérou, en raison de notre grande culture d'architecture en pierre, c'est surtout la pierre qui nous rappelle nos racines maternelles, dont la terre constitue la base physique, mentale et spirituelle. [...] Comme de nombreuses cultures qui accordent une importance particulière à la terre, la culture andine a été persécutée, et en est presque arrivée au bord de l'extinction. Aujourd'hui cette persécution se poursuit d'une façon plus subtile et cela a malheureusement empêché nombre d'entre nous de voir la richesse de la culture précolombienne et du quechua que les Andins eux-mêmes cachent, honteux. Or la cosmovision qu'a créée la culture précolombienne se perpétue dans une pratique mystique et animique qui confère une conscience inhérente au cosmos, à la terre et à tout ce qui en naît. (Mutal 2007, p. 114)

La fascination pour l'ésotérisme andin n'est pas nouvelle mais elle est aujourd'hui mise au service de la mémoire de la guerre. Il s'agit pour les militants des droits humains de susciter un regain d'intérêt pour la sagesse ancestrale indigène. Vanter les mérites spirituels des victimes andines est une manière de les montrer sous un jour plus complaisant, mais tait la nature politique et fratricide du conflit armé dans les campagnes. En effet, adopter une perspective compassionnelle qui fait fi du caractère complexe du déroulement de la guerre revient à présenter 1'ensemble des paysans andins comme des victimes innocentes. La réinvention de la tradition préhispanique, qu'analyse notamment Antoinette Molinié (2009), doit ici être mise en perspective avec la décontamination de la violence qu'elle permettrait.

L'usage de la figure de la Pachamama s'inscrit dans cette perspective ${ }^{29}$. Dans le contexte agropastoral, elle est considérée comme l'esprit tutélaire de la terre, génératrice des produits du sol et du bétail. Elle reçoit un culte à l'occasion des rites propitiatoires et de fertilité. La sphère intellectuelle de Lima est totalement étrangère à cette forme de religiosité andine. Lika Mutal s'est pourtant inspirée de cette divinité pour réaliser la roche centrale d'où coule de l'eau d'un œil en pierre, à la manière de larmes permanentes. Elle a investi la Pachamama d'un sens nouveau. Pour elle, « la mère pleure en raison de ce que se font ses enfants

29. Le vocable quechua «Pachamama » peut être traduit par « Terre mère ». De manière générale, cette récupération de la Pachamama se retrouve dans les mouvements contemporains d'inspiration New Age. L'auteure du mémorial, en s'appuyant sur cette figure qui « est devenue une des images centrales de ces nouvelles dévotions œcuméniques [...] unificatrice de toutes les puissances originaires » (Galinier et Molinié 2006, p. 107), s'inscrit pleinement dans ce courant mystique. 
les uns aux autres ${ }^{30} »$. Convertie en une sorte de Mère Patrie, la Pachamama sert un objectif bien particulier de promotion de la mémoire du conflit armé et de la culture de la paix. Cependant, cette divinité est montrée sous un jour plus séduisant qu'elle ne l'est en réalité dans sa représentation andine.

Cette image [de la Pachamama] a "pris » si bien dans l'histoire andine qu'y compris les anthropologues font référence à elle, malgré le fait que, en réalité, elle apparaît de rares fois dans les sources préhispaniques et que la Pachamama des Indiens réels, avide de sacrifices, est loin d'être aussi sympathique que ne le proposent certains intellectuels. (Molinié 2009, p. 241, traduction de l'auteur)

La Pachamama est ainsi ce qu'on la fait être. À Lima, la nécessité de réconcilier le pays après la guerre civile et la volonté de construire une figure de victime consensuelle conduisent à la réinterpréter et à la présenter comme un avatar de la Mère Patrie. L'image d'un personnage plus bienveillant qu'il n'apparaît dans la cosmologie locale est construite afin d'en faire un symbole universel. Ce travail de réappropriation de la culture andine conduit ainsi à corriger certains de ses éléments emblématiques pour les incorporer plus facilement dans un discours favorable aux droits humains et à la mémoire du conflit armé. Reste que la pierre du mémorial n'est pas forcément associée à l'idée de la Pachamama pour le commun des visiteurs. En effet, lors d'une visite guidée sur le site du mémorial (organisée chaque $1^{\text {er }}$ samedi du mois depuis 2014), une veuve de guerre originaire des Andes centrales me fit le commentaire suivant:

Maintenant je me sens satisfaite. J'ai vu le nom de mon mari, de ma fille [inscrits sur des galets]. Je me sens satisfaite. Mais, dis-moi, je n'ai pas compris l'histoire de la Pachamama... (Entretien avec Trinidad Deza Tello, Lima, le 3 avril 2014)

Rosario Narváez s'était chargée de la visite guidée et elle avait insisté sur la représentation de la Pachamama. Mais visiblement, quand bien même ce symbole est explicité, cela ne le rend pas beaucoup plus intelligible, y compris auprès des paysans andins comme Trinidad. En effet, il y a un important décalage entre la signification de la Pachamama dans le contexte agro-pastoral andin et l'usage qui en est fait dans le contexte mémoriel liménien, et plus largement dans le contexte politique international ${ }^{31}$. Lucides sur cette distance qui peut exister entre la démarche artistique et la perception de l'œuvre, certains organisateurs

\section{Entretien avec Lika Mutal réalisé à Lima le 26 août 2009.}

31. Au-delà de la popularité de la Pachamama dans les milieux altermondialistes et écologistes, c'est à la politisation de cette divinité dans plusieurs pays andins que l'on pense. Le président de la Bolivie, Evo Morales, en a été un promoteur décisif en la remerciant de sa victoire au moment de son investiture (Alvizuri 2009). La notion de Pachamama figure aussi dans la nouvelle Constitution adoptée le 28 septembre 2008 en Équateur. S'exportant au-delà des pays andins, la Pachamama est également devenue une ressource économique importante permettant d'attirer des visiteurs en quête de diversité culturelle dans le nordouest argentin (Boullosa-Jolly 2010). 
des activités commémoratives témoignent eux-mêmes d'une réalité vécue par les visiteurs en décalage avec la conceptualisation de ce lieu de mémoire ${ }^{32}$. Une partie d'entre eux est donc consciente des difficultés qu'il y a à représenter la mémoire de la guerre et du fossé que peut engendrer une mémoire trop intellectualisée. Trinidad, âgée de cinquante-six ans, a vécu la grande majorité de sa vie dans la communauté paysanne de Toraya dans les Andes d'Apurímac et est profondément catholique. C'est la Vierge Marie qu'elle invoque pour rendre hommage à ses proches disparus, pas la Pachamama ${ }^{33}$. Si, aux yeux de cette femme, l'importance de voir les noms de ses proches dans un lieu central de la capitale est indéniable, certains des symboles mis en scène restent largement étrangers et énigmatiques.

L'artiste Ana Correa, membre de la troupe de théâtre Yuyachkani ${ }^{34}$, a aussi contribué à élaborer une image archétypique du rapport au surnaturel dans les Andes comme point de départ aux représentations scéniques du mémorial.

L'Eil-qui-pleure peut être considéré comme les trois mondes de la cosmovision andine. Au centre, la Terre Mère qui pleure fait référence à l'uqu pacha [elle m'écrit ce terme sur mon carnet et dessine un premier cercle], le monde d'en bas, des ancêtres. C'est la commémoration des morts. Ici, [en me dessinant un second cercle autour du premier], c'est le kay pacha, ce monde, le monde des vivants. Et autour [elle dessine un troisième cercle] se trouve le hanaq pacha, le monde d'en haut, le monde des dieux. J'ai pensé utiliser l'espace comme ça, donc [sur cette base] on a commencé à rechercher des comportements et des mouvements. On recherchait d'autres types de connexions qui ne soient pas les plus évidentes, comme cela s'est fait durant toutes ces années. (Entretien avec Ana Correa, Lima, le 19 juin 2009)

32. «Moi je crois que Lika a souhaité transmettre une signification de la pierre. [Mais] Je crois que ce sens n'est pas toujours compris. Je ne crois pas que le message le mieux compris soit l'image de la mère qui pleure parce que de l'eau ruisselle comme une larme permanente. Je ne crois pas que ce soit un message dont, quand quelqu'un le voit [le mémorial], il comprenne "il est en train de pleurer". Quand le visiteur sait qu'il s'appelle L'Eil-qui-pleure, oui, il peut comprendre le concept de douleur qui est exprimé. Mais le concept de "la Terre Mère, représentée à travers la pierre, qui pleure pour ses enfants qui se font du mal les uns aux autres", je ne crois pas que ce soit si facilement compréhensible pour les gens. Je crois qu'ils comprennent le concept de douleur immédiat, mais "la Terre Mère, qui...", je ne crois pas que ce soit compréhensible pour tout le monde » (Rosario Narváez Vargas, Lima, le 26 août 2009).

33. Cette divinité peut en revanche être mobilisée dans un contexte agropastoral pour favoriser la fertilité du bétail et les bonnes récoltes. Par ailleurs, sur l'évolution du rapport aux morts et à l'environnement naturel sous l'effet de la guerre au Pérou, voir Delacroix 2016c, p. 229-269.

34. Les représentations de Yuyachkani ont régulièrement accompagné les cérémonies commémoratives réalisées sur le mémorial. Concevant le théâtre comme « une action politique et une recherche de la culture », Yuyachkani «s'engage dans des actions pour les droits des citoyens ». Le nom de la troupe est en quechua et signifie « je me souviens ». Certains membres de cette troupe de théâtre liménienne, dont Ana Correa, ont accompagné les audiences publiques réalisées dans le cadre des investigations de la CVR à travers des performances sur le thème de la mémoire de la guerre, réalisées dans de nombreuses villes du pays. 
Les « Andins » sont ainsi quasi exclusivement envisagés par les «nonAndins » à travers le prisme de leur spiritualité et de leur cosmologie, alors même que ces dimensions ne constituent qu'un fragment des éléments organisateurs de leur vie sociale. En outre, celles-ci font l'objet d'interprétations souvent simplificatrices, voire erronées, comme cela est le cas de la notion d'inframonde ${ }^{35}$. Pour autant, lorsqu'ils décident de reproduire dans leur communauté une réplique de L'Eil-qui-pleure pour rendre hommage aux leurs et être visibles aux yeux des ONG des droits humains, ils s'inspirent du mémorial le plus emblématique du pays. Ceci surtout en raison de la valeur qui lui est attribuée au sein des mouvements des droits humains. Toute sa symbolique n'est néanmoins pas reprise. Dans la communauté andine de Llinque, en Apurímac, où a été inauguré le 4 juillet 2008 un EEil-qui-pleure à une échelle réduite ${ }^{36}$, la pierre du monument ne représente pas la Pachamama mais la pierre des grottes où les paysans se sont réfugiés et où ils dormaient fréquemment durant la guerre. Par ailleurs, le symbole de la pierre est associé à la pierre de la lapidation, une méthode utilisée par le Sentier Lumineux. Dans les deux cas, la roche extraite de la montagne environnante pour composer le monument est liée à l'expérience de la guerre et a valeur de preuve.

L'armée faisait des allers-retours. Les gens dormaient dans la zone des sommets et dans les grottes. La pierre de L'Eil-qui-pleure est avant tout un témoin pour nous, de toutes nos souffrances, des douleurs et des massacres dont on a souffert. [Il s'arrête.] Les roches nous ont permis de fuir, de nous cacher, mais la pierre a aussi servi au Sentier lumineux pour tuer. Moi je pense que c'est un symbole cette pierre. Elle ne parle pas, c'est un minéral inerte, mais elle a vu les souffrances. (Entretien avec José Pedro Huamani, Llinque, le 17 juillet 2009)

La réplique érigée à Llinque se nourrit et s'oppose à la fois au mémorial liménien ${ }^{37}$. Mais elle permet aussi d'évoquer le passé avec une certaine neutralité politique en présentant « tous les habitants de Llinque » comme d'innocentes victimes. Ceci, dans un contexte d'expectatives très importantes générées par les recommandations de la CVR et les promesses politiques faites aux victimes depuis le conflit armé. Le discours de victimisation qui accompagne l'essor

35. Pour une étude de l'élaboration missionnaire de l'ukhu pacha, voir Estenssoro 2003 et pour une analyse de l'usage contemporain de cette notion dans les récits eschatologiques individuels, voir Robin Azevedo 2004b.

36. Sur les galets qui composent le monument de L'CEil-qui-pleure à Llinque ont été inscrits les noms des vingt victimes originaires de la communauté. Cette monumentalisation de la mémoire fait suite aux ateliers de « sensibilisation aux droits humains » animés dans cette communauté principalement par l'APRODEH et à l'organisation consécutive des paysans dans la revendication de leur statut de victime. Voir Delacroix 2016c.

37. «L'Eil-qui-pleure de Lima a été fait par des professionnels, mais ici sont les faits, ici le sang a coulé », Pablo, Llinque, le 7 juillet 2009. 
du mémorial liménien fait donc l'objet de réappropriation dans les Andes ${ }^{38}$. Les membres des communautés paysannes n'insistent toutefois pas sur une quelconque identité ethnique qui les caractériserait fondamentalement.

Face à l'insistance sur l'andinité des victimes à Lima, on pourrait également objecter que les victimes de cette guerre ne sont pas toutes des paysans indigènes des hautes terres andines. Les populations des villes et de l'Amazonie ont aussi été touchées par le conflit armé. Elles demeurent pourtant peu ou pas représentées lors des commémorations célébrées à L'EEil-qui-pleure. D'autre part, si les paysans des Andes composent, de fait, $79 \%$ des victimes civiles du conflit armé (CVR 2003, p. 316), il serait extrêmement réducteur de croire que tous vivaient dans une situation d'exclusion et de pauvreté comme tendent à le faire croire les discours prononcés lors des cérémonies. Certains étaient de riches négociants en bétail, d'autres appartenaient à des familles de grands propriétaires terriens et l'expérience du service militaire avait, chez certains paysans, contribué à exacerber un sentiment national (Méndez 2006). Pourtant les militants fondent leurs actions sur ce stéréotype du paysan exclu de la société au risque de le renforcer. Le choix et le sens de ce mémorial semblent emblématiques d'une volonté d'évacuer une complexité embarrassante. Soulignons aussi que $26 \%$ des sentiéristes étaient locuteurs quechua selon la CVR ${ }^{39}$. Pourtant, dans ce cas, la langue n'est pas mise en rapport avec l'identité indigène ${ }^{40}$. En outre, quelle place accorder aux paysans andins qui se sont organisés en milices d'auto-défense paysannes, lesquelles ont aussi réglé par le sang de vieux conflits fonciers ${ }^{41}$ ? Ces acteurs aux parcours complexes sont présentés à travers des catégories univoques et exclusives. Tout fonctionne comme si la figure de victime, créée à travers le processus monumental, répondait avant tout aux objectifs sociaux et politiques que se sont fixés les militants : commémorer et réparer les « innocentes victimes indigènes $»^{42}$.

38. De la même manière, un vocabulaire normatif a fait l'objet d'une appropriation et d'une intériorisation de la part des paysans andins. Lorsque, à la fin des années 1990, les ONG commencent à organiser des ateliers de sensibilisation aux droits humains dans les campagnes andines, elles parlent de la guerre et désignent des types d'exactions avec des termes particuliers. Les membres des communautés paysannes vont s'approprier ce vocabulaire afin de verbaliser les différentes violences subies dans le cadre de référence qui est celui des institutions et rendre ainsi audible leurs demandes (Delacroix 2016b).

39. «Violencia y discriminación étnica y racial », in CVR 2003, p. 142-148.

40. Voir Robin Azevedo et Delacroix 2017.

41. Voir notamment Manrique 2007, Degregori 2011 et Robin Azevedo 2014.

42. Sur la façon dont les catégories de victimes et de bourreaux sont pensées de manière fondamentalement hermétique dans le Pérou d'après-guerre, et à partir de critères d'innocence et de culpabilité qui n'acceptent aucun enchevêtrement possible dans la trajectoire d'un seul et même individu, contrairement aux diverses réalités biographiques, voir Manrique 2014, Robin Azevedo 2014 et Delacroix 2016a et b. Sur la mise en avant de leur indigénéité dans la façon même dont la CVR a présenté son rapport final, voir Robin Azevedo et Delacroix 2017. 


\section{Entre ésotérisme et mysticisme : le Qoyllurit'i et les offrandes à la terre}

Si Lika Mutal prête une vision du monde animiste aux paysans des Andes (Mutal 2007, p. 114), elle a elle-même fait sienne cette conception. En réaction aux sculptures d'Europe occidentale qu'elle juge statiques et toutes placées sur un piédestal, elle envisage la pierre d'une manière dynamique, estimant qu'elle bouge très lentement donc qu'elle vit. Ses œuvres ont été largement influencées par les cultures préhispaniques ${ }^{43}$ et une vision animiste du monde minéral. Cette sensibilité de l'artiste ressort particulièrement au sujet de la roche qu'elle a utilisée pour créer L'EEil-qui-pleure.

J'ai des amis qui font partie du groupe d'indigènes que sont les Q'eros, ils vivent à quatre jours de marche de Cuzco. Ils étaient là! Ils sont partis hier! [...] Ils m’ont confié un secret, c'est un savoir pour eux, mais ici les gens ne savent pas, que toutes ces pierres appartiennent à un lignage, au lignage d'un apu. Et L'CEil-qui-pleure qui est dans le Champ-de-Mars est du lignage de l'apu Ausangate qui, pour eux, est un apu tutélaire parce que durant tout le mois de juin on lui dédie une grande cérémonie, le Qoyllurit' $i$ et chaque année des milliers de personnes y vont. [...] Des milliers de personnes dansent, font des offrandes durant trois jours et les plus forts montent au sommet de plus de 6000 mètres d'altitude et apportent des blocs de glaces à la cathédrale de Cuzco. Parce que dans la cathédrale de Cuzco il y a une pierre qu'ils vénèrent. Ce sont des choses qui ne se savent pas, mais qu'ils m’ont confiées. (Entretien avec Lika Mutal, Lima, le 27 août 2009)

Ce témoignage permet de comprendre l'image sur laquelle s'ouvre le courtmétrage dédié à la promotion du mémorial de L'CEil-qui-pleure et dont Mutal a assuré la direction scientifique. Il s'agit d'une photo du pèlerinage du Qoyllurit'i à travers un sommet enneigé ${ }^{44}$. La présentation virtuelle du mémorial est accompagnée d'un cantique en quechua interprété par des artistes de Yuyachkani et la première image de l'Ausangate est suivie d'un gros plan sur l'œil incrusté dans la roche. Une continuité est ainsi instituée entre la cérémonie qui se déroule dans les Andes de Cuzco et le mémorial du parc du Champ-de-Mars. Pour Lika Mutal et selon ses amis Q'eros, la pierre de L'CEil-qui-pleure appartient au lignage de l'Ausangate qui est « le » lieu par excellence des dévotions telluriques et astronomiques au Pérou ${ }^{45}$. Elle a pourtant trouvé la pierre sur la côte Nord péruvienne (dans la Bahía de Independencia à Paracas), mais « peu importe d'où elle provient » garantit-elle lors d'une conversation que nous avons eue le 27 août 2009. Cette incohérence qui ne préoccupe guère l'artiste la

43. Elle a par exemple créé des séries d'animaux mythiques (Pacáric 1974-1975 et Pukará 1980) et une représentation des quipus (Quipu 1974). Voir Boyens 1986.

44. Cet important culte du Christ miraculeux de Qoyllurit'i est célébré à 4600 mètres d'altitude au pied du mont Ausangate dans la région du Cuzco, quelques jours avant la fête du Corpus Christi qui se déroule à Cuzco.

45. Voir notamment Deborah Poole 1988. 
rapproche une fois de plus des tenants du néo-indianisme qui ne s'attachent pas à la véracité de leur propos (Galinier et Molinié 2006). Les Q'eros qu'évoque l'artiste sont d'ailleurs emblématiques de ce mouvement qui émerge au milieu $\mathrm{du} \mathrm{XX}^{\mathrm{e}}$ siècle et sera ultérieurement repris par les New Agers. Convertis en spécialistes religieux, les Q'eros offrent leurs services dans des hôtels de luxe (Molinié 2009, p. 253) et un incaïsme congénital leur est attribué, avec toute la magnificence que cela est censé leur conférer. De même, la fascination de Lika Mutal pour la dimension occulte du savoir des Q'eros et les énergies telluriques des Andes est adossée à une idée de pureté de la culture andine perçue comme une actualisation du passé préhispanique.

Les Q'eros disent être les descendants directs des Incas et ils en ont encore les coutumes. Récemment, en 1950, ils ont rejoint la civilisation. Mais ils sont ravissants tu sais, ils composent leurs tissus et tout, ils sont très beaux. Regarde, ils m'ont donné un tissu, regarde cette merveille! Et eux n'ont pas changé leurs motifs, parce que beaucoup ont changé pour vendre. (Lika Mutal, Lima, le 27 août 2009)

Aussi, inscrit-elle sa pratique mystique et animiste au cœur de sa geste artistique. Au-delà de la figure de la Pachamama, elle articule une kyrielle d'éléments cosmologiques afin de transformer son œuvre en un véritable centre tellurique.

Le dessin [autour du mémorial] est basé sur une étoile à treize pointes. Chiffre qui fait référence aux phases lunaires. Il s'agit d'un labyrinthe dédié à la terre mère, à la lune, à la féminité, à la maternité, le don de la vie contre la masculinité qui devrait protéger la vie, mais qui, dans ce cas, l'a détruite. Le mémorial est orienté vers le Sud. Le Sud c'est le feu, le soleil, il y a donc un mariage entre les forces. Tous les éléments sont là. La pierre regarde vers [la constellation de] la Croix du sud qui parfois, la nuit, quand elle apparaît dans le ciel, se reflète dans l'eau ${ }^{46}$.

Pour l'auteure, une médiation avec le cosmos est permise grâce à la manière dont elle a conçu le lieu. Ce discours est relayé par la presse péruvienne et diffusé par diverses organisations en faveur des droits humains, contribuant ainsi à construire autour de ce mémorial un discours " mystique-ésotérique ${ }^{47}$ caractéristique du New Age. L'CEil-qui-pleure offre en cela un exemple original d'usage d'une ritualité New Age dans un contexte de revendications politiques et sociales portées par les ONG des droits humains qui exigent la vérité, la

46. Lika Mutal citée par Roxana Chirinos, « Conversación con Lika Mutal », paru sur le site agenciaperu.com, traduction de l'auteur, http://www.verdadyreconciliacionperu.com/ admin/files/articulos/123_digitalizacion.pdf, consulté le 25/10/2017.

47. J'emprunte à Françoise Champion et Martine Cohen les termes génériques de « nébuleuse mystique-ésotérique » au sens d' « ensemble hétérogène de groupes, ou plutôt de réseaux, souvent lâches, pouvant se rattacher plus ou moins explicitement à des traditions religieuses constituées - religions orientales (hindouisme, bouddhisme) ou plus "exotiques" (notamment au chamanisme) - et pouvant aussi réactiver diverses pratiques ésotériques, tout particulièrement le tarot et l'astrologie » (Champion et Cohen 1993). 
justice et l'exécution des programmes de réparations aux victimes. Or c'est bien parce qu'ils sont animés d'un souci d'amélioration des conditions de vie de la paysannerie andine et de respect de leurs droits fondamentaux que ces travailleurs d'associations nationales, majoritairement issus de l'intelligentsia du pays, ne peuvent être uniformément qualifiés de néo-Indiens ${ }^{48}$. Bien qu'individuellement certains militants cumulent les critères d'appartenance à ce mouvement idéologique et rituel, il n'en reste pas moins que nous sommes face à une cosmologie mystique mise au service d'un intérêt politique supérieur: la cause des victimes. Déjà mobilisée par les mouvements écologiques, voilà la « spiritualité andine » transformée en outil de sensibilisation aux droits humains. Dans ce complexe politico-religieux, l'exigence de judiciarisation des coupables d'atrocités peut être soutenue par des offrandes à la terre ${ }^{49}$.

Le 28 août 2009, lors de la cérémonie d'anniversaire du rapport final de la CVR, on pouvait voir disposés sur le pago a la tierra élevé sur le mémorial des pommes de terre, des feuilles de coca, des gâteaux, des bonbons, deux bouteilles d'alcool, de la sauge, des haricots et du riz présentés dans des pots de terre, une assiette avec du maïs et enfin une balance portant une bougie rouge et une autre blanche, aux couleurs du drapeau national péruvien. Par ailleurs, Rosario Narváez Vargas, principale organisatrice du déroulement de la cérémonie du 28 août, avait suggéré aux familles d'ajouter des éléments permettant d'identifier leurs parents disparus. Certains ont ainsi amené l'uniforme militaire d'un parent tué pendant la guerre ou encore du pain et du chocolat, aliments particulièrement appréciés par la personne de son vivant. Le résultat mêlait les caractéristiques d'un autel funéraire et d'une offrande à la terre. Selon Rosario, cette utilisation du rapport au divin dans les Andes participe d'un mode de diffusion et d'appréhension de cette culture « autre » à Lima.

Sans parler spécifiquement de L'Eil-qui-pleure, il y a diverses initiatives ailleurs qui utilisent certains éléments de la cosmogonie andine dans un sens positif, avec la bonne intention de se rappeler, de récupérer une identité, que les gens sachent, que ça se diffuse. Le fait que les gens de Lima s'emparent [de ces initiatives] et parfois se l'approprient davantage que ne le feraient les propres andins; je l'ai vu dans diverses activités. [...] Je crois qu'à Lima c'est un peu imposé, importé,

48. Le mouvement néo-indien n'est ni indianiste ni indigéniste comme le soulignent Galinier et Molinié (2006, p. 19). Les néo-Indiens ignorent les caractéristiques sociales des populations desquelles ils disent puiser «l'énergie ». En outre, la première ébauche de la question du cadre idéologique et rituel qui sous-tend les commémorations de la guerre dans la capitale, proposée dans cet article, nécessiterait un travail systématique auprès des militants des droits humains afin explorer plus finement leurs positionnements spirituels et politiques.

49. Dans un contexte agropastoral, ces offrandes ont pour objectif principal de séduire les êtres tutélaires andins [en rassasiant] leur appétit pour la nourriture rituelle (Fernández Juárez 1997). Le pago ou despacho « consiste à disposer, selon un code très précis, divers ingrédients dans un espace rituel limité par un tissu consacré (mesa) »(Galinier et Molinié 2006). 
mais ce n'est pas une mauvaise chose que ça soit comme ça. Même si je crois que cela n'a pas de sens pour tous, ce n'est pas une mauvaise chose que ces visions du monde soient reprises parce que je crois, et je le dis haut et fort, qu'elles permettent d'encadrer les actes dans un contexte historique et une réalité culturelle. (Entretien avec Rosario Narváez, Lima, le 26 août 2009)

La militante admet donc le caractère un peu forcé de l'appropriation. Pour autant, elle ne nie pas son efficacité à saisir une « réalité culturelle ». Cette présentation stéréotypée contrarie certains militants qui ne souhaitent pas enfermer ceux qu'ils défendent dans une culture qui est communément perçue comme archaïque par une large frange des Liméniens. Toutefois, en mettant en avant les populations andines, en insistant sur leur mort massive et en valorisant leur « vision du monde », les ONG participent à leur réification et les figent dans une image intemporelle et irréelle. Cette perspective peut être rapprochée des discours indigénistes de défense des populations autochtones dans les années vingt, lesquels ont ensuite été relayés par des organisations internationales et par certains États jusque dans les années soixante-dix (Lavaud et Lestage 2006). D'ailleurs, cet éloge de l'indianité pour servir la mémoire de la guerre s'inscrit dans un même circuit de réciprocité tronquée entre paysans et militants, ici bénéficiaires et pourvoyeurs de l'aide humanitaire et des réparations aux victimes, et se fait au détriment de la qualité de sujet politique des personnes dont le souvenir est célébré.

\section{Conclusion}

Le devoir de mémoire partagé parmi les militants des droits humains et une partie de l'intelligentsia de gauche est étroitement lié à un sentiment de dette morale envers les populations andines quechuaphones qui ont été les plus affectées par le conflit armé, et ce au détriment des autres morts de la guerre qui sont rendus moins visibles. Cette position a pour conséquence la valorisation d'une culture andine homogène de la part des personnes qui se considèrent comme étant extérieures à elle et l'envisagent de manière dépolitisée. Si les organisateurs des activités qui se déroulent sur le site de L'Eil-qui-pleure de Lima sont animés d'une volonté de reconnaissance et d'intégration sociale des victimes, ils continuent néanmoins de reproduire un discours sur l'Autre et une division de la société péruvienne que la CVR elle-même avait identifiés comme source de la violence ${ }^{50}$. La riche vision du monde qui est prêtée aux paysans andins vient appuyer leur innocence et ces catégorisations réductrices

50. Poole et Rojas-Perez (2005) expliquent que cela est également le cas de l'exposition photographique Yuyanapaq, pourtant soutenue par la CVR, mais qui contribue à entériner toute une série de perceptions visuelles stéréotypées à l'égard des paysans andins. 
empêchent le développement d'une critique de la violence au sein même de la paysannerie andine durant la guerre.

Le mémorial tout entier est tourné vers une spiritualisation des victimes, qui tend à les présenter comme absolument innocentes. La figure de la mère, véhiculée à travers la Pachamama, permet de s'éloigner d'une virilité supposée agressive; tandis que l'accent mis sur la cosmologie des victimes et non sur leur pensée articulée gomme le fait qu'elles aient pu combattre à un moment particulier de la guerre. En effet, L'Eil-qui-pleure a été pensé comme un support transcendant les choix politiques et permettant de rappeler la globalisation du phénomène de violence à une échelle supérieure à celle de l'individu. Le Pérou a perdu près de 70000 de ses citoyens durant cette guerre et c'est en mémoire de chacun d'eux que l'eau coule de l'œil du mémorial, symbolisant les larmes de la communauté nationale face à leur perte. La Terre Mère devient la Mère Patrie. Tous les galets sont soudés dans une semelle de fondation commune, incarnant un même conflit intra-national. Ce discours dépolitisé, au moins dans sa conception initiale, et ce processus d'ethnicisation des morts échouent à faire de ce lieu de mémoire un lieu où l'histoire récente serait traitée de façon complexe. Y prime une instrumentalisation du passé préhispanique et de certaines formes du rapport au religieux dans les Andes pour servir des objectifs de revendication des droits humains.

L'émergence de ce nouveau type de ritualité politique mise au service de la mémoire de la guerre se retrouve également au Guatemala. Bien que le déchaînement des violences réponde à des contextes historiques et politiques différents, les populations indigènes ont été les plus touchées par les récents conflits armés internes guatémaltèque et péruvien. Or, on assiste simultanément à l'essor d'une ethnicisation de la mémoire des violences qui se traduit par la diffusion d'une spiritualité pan-indigène lors des ateliers réalisés par différentes ONG et durant les activités commémoratives. Le projet de construire une réplique de L'Eil-qui-pleure au Guatemala ${ }^{51}$, bien qu'il semble avoir avorté, témoigne de cette transmutabilité de la mémorialisation lorsqu'il s'agit de rendre hommage à une catégorie d'individus dont on présume qu'ils partagent des représentations du monde assez similaires.

En définitive, nous sommes face à un discours victimaire qu'il faut replacer dans divers usages contextuels. Il sert d'abord de support afin de formuler de manière acceptable un certain nombre d'attentes propres aux communautés paysannes sur la scène publique et dans le contexte post-CVR. Cette concentration sur l'image de la victime andine est aussi satisfaisante pour les militants des droits humains qui y puisent la légitimité même de leur action dans les zones rurales du pays, lesquelles étaient considérées, pendant la guerre, comme le

51. Lika Mutal, communication personnelle, le 26 août 2009. 
berceau de la subversion. Enfin, il faut bien considérer que les projecteurs braqués sur une victime dépolitisée offrent aussi à l'État un moyen de désamorcer les potentielles critiques envers l'ordre social et économique profondément inique et discriminatoire qui a constitué un terrain fertile au déploiement du phénomène de violence politique des deux dernières décennies du $\mathrm{Xx}^{\mathrm{e}}$ siècle. *

* Manuscrit reçu en février 2016, accepté pour publication en avril 2017.

\section{Références bibliographiques}

Alvizuri Verushka

2009 La Construcción de la aymaraidad. Una historia de la etnicidad en Bolivia (1952-2006), Editorial El País, La Paz.

Boullosa-Joly Maïté

2010 «Tourisme, patrimonialisation et politique. Un cas d'école: la "Fête nationale de la Pachamama" (Nord-Ouest argentin) », Cahiers des Amériques latines, 65 , p. 103-120.

Boyens José

1986 «La pierre porteuse d'esprit. La sculpture de Lika Mutal», Septentrion, 15 (1), p. 42-47.

BROKAw Galen

2010 A History of the Khipu, Cambridge University Press, Cambridge.

CHAMPIOn Françoise et Martine CoHEN

1993 «Recompositions, décompositions. Le renouveau charismatique et la nébuleuse mystique-ésotérique depuis les années soixante-dix », Le Débat, 75, p. 77-85.

COMISIÓN DE LA VERDAD Y RECONCILIACIÓN (CVR)

2003 Informe final, Lima, http://www.cverdad.org.pe/ifinal/index.php, consulté le $23 / 10 / 2017$.

COMISIÓN PARA EL ESCLARECIMIENTO HISTÓRICO

1999 Guatemala: Memoria del Silencio, Oficina de Servicios para Proyectos de las Naciones Unidas (UNOPS), Ciudad de Guatemala.

Degregori Carlos Iván

2011 Qué difícil es ser Dios. El partido comunista del Perú Sendero Luminoso y el conflicto armado interno en el Perú: 1980-1999, IEP, Lima.

Delacroix Dorothée

2014 «"Somos Peruanos y limpios": discursos y prácticas en torno al monumento "El Ojo que llora" de Llinque, Apurímac », Bulletin de l'Institut français d'études andines, 43 (2), p. 227-244.

2016a «Le terroriste et la victime: deux catégories hermétiques? Complexité des mémoires de la guerre au Pérou à travers l'usage des monuments aux morts », in Clara Duterme, Marion Giraldou et Abigail Mira, Mauvais sujets dans les Amériques. Répression, représentations, discours autour des populations indésirables, Presses universitaires du Midi, Toulouse, p. 61-76. 
Delacroix Dorothée

2016 b « Sortir de prison : revenir de la mort. Marquage du corps et de l'âme à travers l'expérience carcérale au Pérou », in Anne-Marie Losonczy et Valérie Robin Azevedo, Retour des corps, parcours des âmes. Exhumations et deuils collectifs dans le monde hispanophone, Paris, Éditions Pétra, p. 173-201.

2016c De pierres et de larmes. Mémorialisation et discours victimaire dans le Pérou d'après-guerre, Institut universitaire Varenne/LGDJ-Lextenso éditions, Paris.

DRINOT Paulo

2007 «El Ojo que Llora. Las ontologías de la violencia y la opción por la memoria en el Perú », Hueso Huméro, 50, p. 53-74.

EstensSoro Juan Carlos

2003 Del Paganismo a la santidad. La incorporación de los indios del Perú al catolicismo, 1532-1750, IFEA, Lima.

FAVRe Henri

1996 L'Indigénisme, PUF (Que sais-je?), Paris.

2009 «El movimiento indianista: un fenómeno "glocal” », in Valérie Robin Azevedo et Carmen Salazar-Soler (éd.), El regreso de lo indígena. Retos, problemas y perspectivas, Instituto francés de estudios andinos (Actes et mémoires, 26), Lima, p. 29-37.

FERNÁNDEZ Jú́REZ Gerardo

1997 Entre la repugnancia y la seducción. Ofrendas complejas en los Andes del Sur, Centro de Estudios Regionales Andinos Bartolomé de Las Casas (Archivos de historia andina, 24), Cuzco.

Francke Ballve Marfil

1980 «El movimiento indigenista en el Cuzco, 1910-1930 », in Carlos Iván Degregori, Mariano Valderrama, Augusta Alfajeme, Marfil Francke Ballve, Indigenismo, clases sociales y problema nacional. La discusión sobre el "problema indígena" en el Perú, Centro Latinoamericano de Trabajo Social (CELATS), Lima, p. 86-107.

GaLinier Jacques et Antoinette Molinié

2006 Les néo-Indiens. Une religion du III millénaire, Odile Jacob, Paris.

Hite Katherine

2007 " "The Eye that cries": the politics of representing victims in contemporary Peru », A Contra Corriente, 5 (1), p. 108-134.

ITIER César

2010 Les Incas, Les Belles Lettres, Paris.

JALLADE Sébastien

2011 «La réinvention des routes incas; représentations et construction de la mémoire au Pérou (2001-2011) », Droits et cultures, 62, p. 119-137.

Lavaud Jean-Pierre et Françoise Lestage

2006 «L’indianisme en Amérique latine. Historique, réseaux, discours, effets pervers », Esprit, janvier 2006, p. 31-46.

MANRIQue Marie J.

2014 «Generando la inocencia: creación, uso e implicancias de la identidad de "inocente" en los periodos de conflicto y post-conflicto en Perú », Bulletin de l'Institut français d'études andines, 43 (1), p. 53-73. 
MANRiQue Nelson

2007 «Pensamiento, acción y base política del movimiento Sendero Luminoso. La guerra y las repuestas de los comuneros (1964-1983) », in Anne PérotinDumon (dir.), Historizar el pasado vivo en América Latina [en ligne], Universidad Alberto Hurtado, Centro de Etica, [Santiago, Chile], http://www. historizarelpasadovivo.cl/, consulté le 25/10/2017.

Méndez Cecilia

1993 Incas sí, Indios no. Apuntes para el estudio del nacionalismo criollo en el Perú, Instituto de Estudios Peruanos (Documento de trabajo, 56), Lima.

2006 «Las Paradojas del autoritarismo: Ejército, Campesinado y Etnicidad en el Perú: siglos XIX al XXI », Iconos. Revista de Ciencias Sociales, 26, p. 17-34.

Milton Cinthya

2011 «Defacing memory: (Un)tying Peru's memory knots », Memory Studies, 4 (2), p. 190-205.

Molinié Antoinette

2009 «Del Inca nacional a la Internacional Inca », in Valérie Robin Azevedo et Carmen Salazar-Soler (éd.), El regreso de lo indígena, retos, problemas y perspectivas, Instituto Francés de Estudios Andinos (Actes et mémoires, 26), Lima/Centro de Estudios Regionales Andinos Bartolomé de Las Casas (Estudios y debates regionales andinos, 3109), Cuzco.

Mouchard Daniel

2000 «Réconcilier, réprimer: les "années de plomb” en Italie et les transitions démocratiques dans le cône sud latino-américain », Cultures \& Conflits [en ligne], 40, http://conflits.revues.org/479, consulté le 25/10/2017.

Mutal Lika

2007 «La conscience de l'espace. De l'espace rêvé, des pierres sacrées, du munay », Revue du GERFLINT, 2 (« Identité plurielle: regard croisé langue-culturescience »), p. 114-118.

PIEL Jean

1975 Capitalisme agraire au Pérou, Anthropos, Paris.

Poole Deborah

1988 «Entre el milagro y la mercancia: Qoyllu rit’i », Márgenes, 2 (4) p. 101-119.

Poole Deborah et Gerardo RÉniQue

1992 Peru. Time of fear, Latin America Bureau, Londres.

Poole Deborah et Isaís Rojas-Perez

2005 «Memorias de la reconciliación: fotografía y memoria en el Perú de la posguerra », e-misférica, 7 (2), http://hemi.nyu.edu/hemi/es/e-misferica-72/ poolerojas, consulté le 25/10/2017.

RÉNIQUe José Luis

1991 Sueños de la sierra. Cusco en el siglo XX, CEPES, Lima.

Riviale Pascal

2001 « Alcide d'Orbigny, l'homme péruvien et les débuts de l'anthropologie », in Michel Bertrand et Laurent Vidal (dir.), À la redécouverte des Amériques. Les voyageurs européens au siècle des indépendances, Presses universitaires du Mirail (Tempus), Toulouse, p. 37-52. 
Robin Azevedo Valérie

2004a «Indiens, Quechuas ou Paysans? À propos des communautés quechuaphones des Andes sud-péruviennes ", Amérique Latine Histoire et Mémoire. Les Cahiers ALHIM, 10, p. 35-44, https://alhim.revues.org/98, consulté le 25/10/2017.

2004b « La divine comédie dans les Andes ou les tribulations du mort vers son voyage vers l'au-delà », Journal de la Société des américanistes, 90 (1), p. 143-181.

2014 « ¿Verdugo, héroe o víctima? Memorias de un rondero campesino ayacuchano (Perú) », Bulletin de l'Institut français d'études andines, 43 (2), p. 245-264.

Robin Azevedo Valérie et Dorothée Delacroix

2017 «Categorización étnica, conflicto armado interno y reparaciones simbólicas en el Perú post-Comisión de la Verdad y Reconciliación », Nuevos mundos mundos nuevos [en ligne], rubrique « Débats », http://journals.openedition. org/nuevomundo/71688, consulté le 11/12/2017.

SALOMON Franck

2004 The Cord Keepers: Khipus and Cultural Life in a Peruvian Village, Duke University Press, Durham.

ThIESSE Anne-Marie

1999 La création des identités nationales, Éditions du Seuil, Paris.

Todorov Tzvetan

1993 « La mémoire et ses abus », Esprit, 193, p. 34-44.

VANTHUYNe Karine

2014 La présence d'un passé de violence. Mémoires et identités autochtones dans le Guatemala de l'après-génocide, Presses de l'université de Laval, Laval.

WALKER Charles

2015 La rebelión de Tupac Amaru, Instituto de Estudios Peruanos, Lima. 